\title{
Perception paysanne et dénomination des plantes vasculaires en société mossé: cas des régions du nord, du centre-nord, du centre et du Plateau Central du Burkina Faso (Afrique de l'Ouest)
}

\author{
Received: 2017-08-12; revised: 2017-09-30; accepted: 2017-10-22
}

\begin{abstract}
${ }^{1}$ Salfo Savadogo, ${ }^{2}$ Issaka Ouedraogo, ${ }^{3}$ Adjima Thiombiano
${ }^{1}$ Département Substances Naturelles (DSN), Institut de Recherche en Sciences Appliquées et Technologies (IRSAT), Centre National de la Recherche Scientifique et Technologique (CNRST), 03 BP 7047 Ouagadougou 03; Tél: (226) 50356031/ (226) 78148774/ (226) 76091008; Email: asalfosava@yahoo.fr/salfosava@gmail.com

${ }^{2}$ Institut des Sciences (IDS), 01 BP 1757 Ouagadougou 01 Burkina Faso

${ }^{3}$ Laboratoire de Biologie et Ecologie Végétales (LaBEV), Unité de Formation et de Recherche en Sciences de la Vie et de la Terre (UFR/SVT), Université Ouaga I Professeur Joseph Ki Zerbo (UO I/Pr JKZ), 09 BP 848 Ouagadougou 09 Burkina Faso
\end{abstract}

\begin{abstract}
Résumé: Cette étude analyse les stratégies locales de dénomination des espèces végétales par les Mossé des régions du nord, du centre nord, du centre et du Plateau Central du Burkina Faso et leurs perceptions des plantes. A travers des interviews semi directes auprès de 1437 personnes âgées d'au moins 60 ans et des jeunes de moins de 40 ans des différentes localités, l'étude a pu montrer les critères de dénomination, les conceptions que les populations ont des espèces végétales ainsi que l'impact de ces connaissances dans la conservation de la phytodiversité. 72 espèces au total ont été décrites. Elles sont réparties en 51 genres et 29 familles. Les familles dominantes sont les Commelinaceae et les Fabaceae-Mimosoideae. Dans la taxonomie locale faite sur les plantes en milieu rural Mossé, 16 critères sont utilisés. Les critères les plus cités par la population sont l'usage fait de la plante $(94 \%)$, le mysticisme lié à l'espèce $(86 \%)$, l'écologie ou le milieu de vie de l'espèce (83\%), la dualité mâle/femelle $(83 \%)$, la couleur des organes ou parties de la plante $(81 \%)$, l'origine de la plante $(80 \%)$, la morphologie foliaire $(76 \%)$, la présence d'organes saillants sur la plante $(75 \%)$ et le mode de dissémination des fruits ou des graines $(74$ $\%)$. Les noms botaniques attribués aux plantes varient d'une région à une autre. Les populations ont des perceptions vis-à-vis de nombreuses espèces. Ainsi, les espèces comme Stereospermum kunthianum, Calotropis procera, Ozoroa insignis, Faidherbia albida, Maytenus senegalensis et Biophytum umbraculum sont frappées de mysticisme. Elles sont toutes craintes par les populations et sont dans certaines localités à l'abri d'exploitations multiformes humaines. Cela contribue à une meilleure conservation de la biodiversité.
\end{abstract}

Mots clés: ethnobotanique, noms vernaculaires, mooré, dénomination, biodiversité, interview

\section{PEASANT PERCEPTION AND VASCULAR PLANTS DENOMINATION IN MOSSÉ SOCIETY: A CASE STUDY IN NORTH, NORTh-Central, Central ANd Plateau Central Regions Of BuRkina Faso (West Africa)}

Summary: This study analyzes local strategies for naming plants by the Mossé of North, North-central, Central and Plateau Central regions in Burkina Faso and their perceptions of the plants. Through semi-structured interviews with 1437 people aged at least 60 years and young people under 40 of different localities, the study has allowed to show the naming criteria, the conceptions that populations have of plant species and the impact of this knowledge in the conservation of plant diversity. 72 species in total were described, belonging to 51 genera and 29 families. The most dominant families are Commelinaceae and Fabaceae-Mimosoideae. In the local taxonomy of plants in Mossé rural areas, 16 criteria are used. The most cited criteria are the use of the plant (94\%), the mysticism related to the species $(86 \%)$, the ecology or the living environment of the species $(83 \%)$, male/female duality $(83 \%)$, color of organs or parts of the plant $(81 \%)$, plant origin $(80 \%)$, leaf morphology $(76 \%)$, the presence of prominent organs on the plant (75\%) and the mode of dissemination of fruits or seeds $(74 \%)$. The botanical names vary from one region to another. People have perceptions of many species. Thus, species such as Stereospermum kunthianum, Calotropis procera, Ozoroa insignis, Faidherbia albida, Maytenus senegalensis and Biophytum umbraculum are mystical. They are all feared by the local people and are in certain localities exempt from multiform human exploitation. This contributes to a better conservation of biodiversity.

Key words: ethnobotany, plant species, vernacular names, mooré, denomination, biodiversity, interview

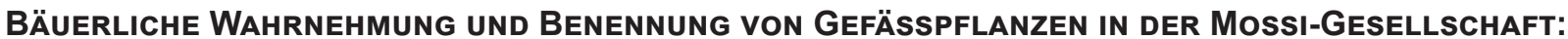 Eine Fallstudie in den Zentralen Regionen Nord-, NoRd-Zentral- Und Zentral Und Zentralpla- TEAU IN BURKINA FASO (WESTAFRIKA)}

Zusammenfassung: Diese Studie analysiert die Wahrnehmung und lokale Strategien für die Benennung von Pflanzen durch die Mossi der Nord-, Nord-Zentral-, Zentral- und Zentral- und Zentralregion in Burkina Faso. Durch semi-strukturierte Interviews mit 1437 Personen im Alter von mindestens 60 Jahren und Jugendlichen unter 40 Jahren an verschiedenen Orten konnte die Studie die Benennungskriterien, die Vorstellungen von Pflanzenarten und die Auswirkungen dieses Wissens auf die Erhaltung der Pflanzenvielfalt zeigen. Insgesamt wurden 72 Arten aus 51 Gattungen und 29 Familien erfasst, wobei Commelinaceae und Fabaceae-Mimosoideae die dominantesten Familien sind. In der lokalen Taxonomie von Pflanzen werden in 
ländlichen Gebieten von den Mossi 16 Kriterien verwendet. Die am häufigsten genannten Kriterien sind: Verwendung (94 \%), mit der Art verbundene Mystik (86 \%), Ökologie oder das Lebensraum (83 \%), Dualität männlich/weiblich (83 \%), Farbe von Organen oder Teilen (81 \%), Herkunft $(80 \%)$, Blattmorphologie $(76 \%)$, Vorhandensein prominenter Organe $(75 \%)$ und Verbreitung von Früchten oder Samen (74 \%). Die botanischen Namen variieren von Region zu Region. Die Menschen nehmen die Arten unter diversen Aspekten wahr. Beispielsweise sind Stereospermum kunthianum, Calotropis procera, Ozoroa insignis, Faidherbia albida, Maytenus senegalensis und Biophytum umbraculum mystisch. Sie alle werden von der lokalen Bevölkerung gefürchtet und daher zumindest an bestimmten Orten von jeglicher Nutzung ausgenommen, was für die Erhaltung der Biodiversität förderlich ist.

Schlagworte: Ethnobotanik. Vernakulärnamen, Mooré, Benennungs-Konzepte, Biodiversität, Interview

\section{INTRODUCTION}

Les peuples autochtones, les communautés locales et leurs savoirs locaux jouent un rôle important dans la conservation de la biodiversité. Ils interviennent dans plusieurs domaines notamment agricoles, sanitaire, culturel, botanique, géographique, etc. Selon certains auteurs, la collecte des données ethnobotaniques permet de mettre en évidence des savoirs sur la biologie et l'écologie des espèces d'une grande importance (FOALE 1998; NeIS et al. 1999; JOHANNES et al. 2000; Obura 2001; Sabetian 2002; Duncan 2005). Ces savoirs traditionnels constituent une accumulation de connaissances, de savoir-faire, de pratiques et de représentations maintenus, transmis et développés par les peuples dont l'histoire se fond dans l'environnement naturel (KYINDOU 2000; YANN Le GoATER 2007). Cette collection sophistiquée de compréhensions, interprétations et significations fait partie d'un ensemble culturel encore plus complexe qui comprend le langage, les rites, la spiritualité et la cosmogonie (YANn Le Goater 2007).

Ces savoirs locaux constituent une richesse du point de vue de la diversité biologique. Quatre-vingt pourcent (80\%) de la population mondiale continue d'user des savoirs et méthodes traditionnelles pour se nourrir et se soigner (KHOR 2002). C'est pourquoi EsoH (2003) estime que: «la connaissance de l'autre, de sa culture, des valeurs auxquelles il s'identifie et croit, est le point de départ pour toute politique de gestion intégrée des forêts tropicales». Abondant dans le même sens, KYINDOU (2000) argumente que l'accès à l'information sur l'environnement biophysique, et particulièrement celle qui touche à la biodiversité animale ou végétale ainsi que son usage, reste influencé par un certain nombre d'éléments liés à la culture des populations qui ont un lien direct avec cet environnement. Ces éléments incluent les savoirs, les croyances, le droit et les coutumes d'une société donnée.

Cependant, ces savoirs locaux connaissent de nos jours d'importantes menaces perpétrées par le modernisme, la frange jeune de la population (jeune génération) et les religions importées (islam et christianisme). Selon SAVADOGO et al. (2011) et SAVADOGO (2013), ces religions monothéistes ou importées constituent une menace pour certaines valeurs sociales jadis entretenues par les religions traditionnelles. Parmi elles, on peut citer les règles et les principes qui régissent la gestion des bois sacrés, les perceptions positives qu'ont certaines populations vis-à-vis des bois sacrés et des espèces végétales sacrées (SAvadogo 2013). Comme le relèvent BoKDAM et Droogers (1975), la disparition des plantes sauvages sous l'influence de la modernisation conduit à la perte des connaissances relatives à leurs usages, leurs noms et leurs étymologies. Au regard de ces menaces sur l'environnement, et vue l'importance des savoirs tradi- tionnels dans la survie de cet environnement, il est impérieux de disposer des connaissances précises sur ces savoirs locaux afin de les valoriser. La société Moagha a une longue tradition de pratique animiste qui tend à régresser avec la poussée des religions importées comme l'islam et le christianisme (SAVAdogo et al. 2011; SAVAdogo 2013). Par ailleurs, les Mossé constituent le groupe ethnique majoritaire au Burkina Faso (50\% de la population). Ils sont fortement concentrés dans les régions du nord, du centre-nord, du Plateau Central et du centre. Malgré la pression de certains facteurs sociaux sur la tradition, les pratiques culturelles sont toujours maintenues dans ces régions par les dignitaires de la tradition (autorités coutumières, gardiens de fétiches, notables, ...).

C'est pourquoi cette étude a été initiée dans ces régions afin de rendre compte de l'état actuel des connaissances locales sur les espèces végétales. Elle a pour objectif de savoir les connaissances locales sur le mode d'organisation et de catégorisation de la flore afin de mettre en évidence un trésor de savoirs locaux sur la biologie et l'écologie des espèces. De façon spécifique, cette étude vise à:

- identifier les critères utilisés par les populations locales dans la dénomination et la classification des espèces végétales locales;

- répertorier les différentes variations des noms vernaculaires suivants les localités (les synonymies et les homonymies);

- appréhender les perceptions paysannes sur ces espèces;

- apprécier l'impact des perceptions paysannes et de l'ethnotaxonomie sur la conservation des espèces.

\section{MÉTHOdes}

\subsection{Milieu d'étude}

Le Burkina Faso est situé en Afrique de 1'ouest, dans la boucle du Niger, entre les latitudes $09^{\circ} 02^{\prime}$ ' et $15^{\circ} 05$ Nord et les longitudes $02^{\circ} 02^{\prime}$ Est et $05^{\circ} 03^{\prime}$ Ouest (Fig.1). Le climat est de type tropical et se caractérise par l'alternance d'une longue saison sèche et d'une courte saison des pluies de mai/ juin à septembre, avec de très fortes variations interannuelles de la pluviométrie. Le découpage climatique permet de distinguer trois zones principales (Ministère de l'Économie et du Développement 2006):

- la zone soudanienne qui occupe toute la portion sud du pays avec une saison de pluies qui dure six mois et des pluviométries pouvant aller jusqu'à $1200 \mathrm{~mm}$ par an;

- la zone soudano-sahélienne, comprise entre les isohyètes $600 \mathrm{~mm}$ et $900 \mathrm{~mm}$, s'étale sur tout le centre du 
pays et constitue la région climatique la plus vaste du Burkina Faso;

- la zone sahélienne: elle représente $25 \%$ de la superficie du pays et est limitée au sud par l'isohyète $600 \mathrm{~mm}$.

Le pays compte plus de 17 millions d'habitants, inégalement répartis et représentant une soixantaine de groupes ethniques dont les principaux sont: les Mossé (50\%), les Bobo (9\%), les Peulh (9\%), les Gourmantché (7\%) et les Gourounsi (6\%) (YAHMED 2006). Chaque groupe se distingue des autres par la langue, les coutumes, l'organisation sociale, l'habitat, le mode de vie, etc.

L'étude a été réalisée dans les régions du nord, du centrenord, du centre et du Plateau Central du Burkina Faso (Fig. 1); les mossé qui constituent le groupe ethnique majoritaire au Burkina Faso y sont fortement concentrés. La société moagha (singulier de mossé) a été choisie non seulement à cause de sa forte représentativité à l'échelle nationale mais aussi par ce qu'elle a longtemps conservé des pratiques ancestrales. La langue des Mossé est le Mooré. Cependant, plusieurs dialectes sont parlés par les Mossé dans la zone d'étude. Ces dialectes varient du nord jusqu'au centre du pays, en passant par le Plateau Central. Les Mossé pratiquent les trois principales religions présentes au Burkina Faso à savoir l'islam, le christianisme et l'animisme. Mais, d'une manière générale, les Mossé tout comme les autres ethnies pratiquent le syncrétisme religieux. Qu'ils soient musulmans ou chrétiens, ils font souvent recours aux pratiques de la religion traditionnelle.

\subsection{Collecte des données}

La collecte des données a consisté en une enquête basée sur des interviews semi structurées en groupe de 15 personnes environ. Au cours d'une interview en groupe, les réponses peuvent être corrigées et réajustées par des membres du groupe qui constateraient des imprécisions ou des erreurs (SAVAdogo 2013).

Les travaux ont été menés dans quatre-vingt-dix (90) villages appartenant à sept (7) provinces et trois (3) régions. Le choix des villages a été centré sur des terroirs Mossé. Les différentes localités ont été choisies sur la base de la vivacité de la tradition et des coutumes (ampleur des sacrifices qui y sont liés, capacités des communautés à maintenir les traditions existantes, constances des évènements rituels de nature à encourager les cultures et les traditions,...). Ce choix a été guidé par les autorités coutumières, les personnes d'âges avancés (au moins 60 ans) et les jeunes de moins de 40 ans. Ainsi, après une première interview dans une localité orientée par un guide de terrain autochtone de ladite localité,

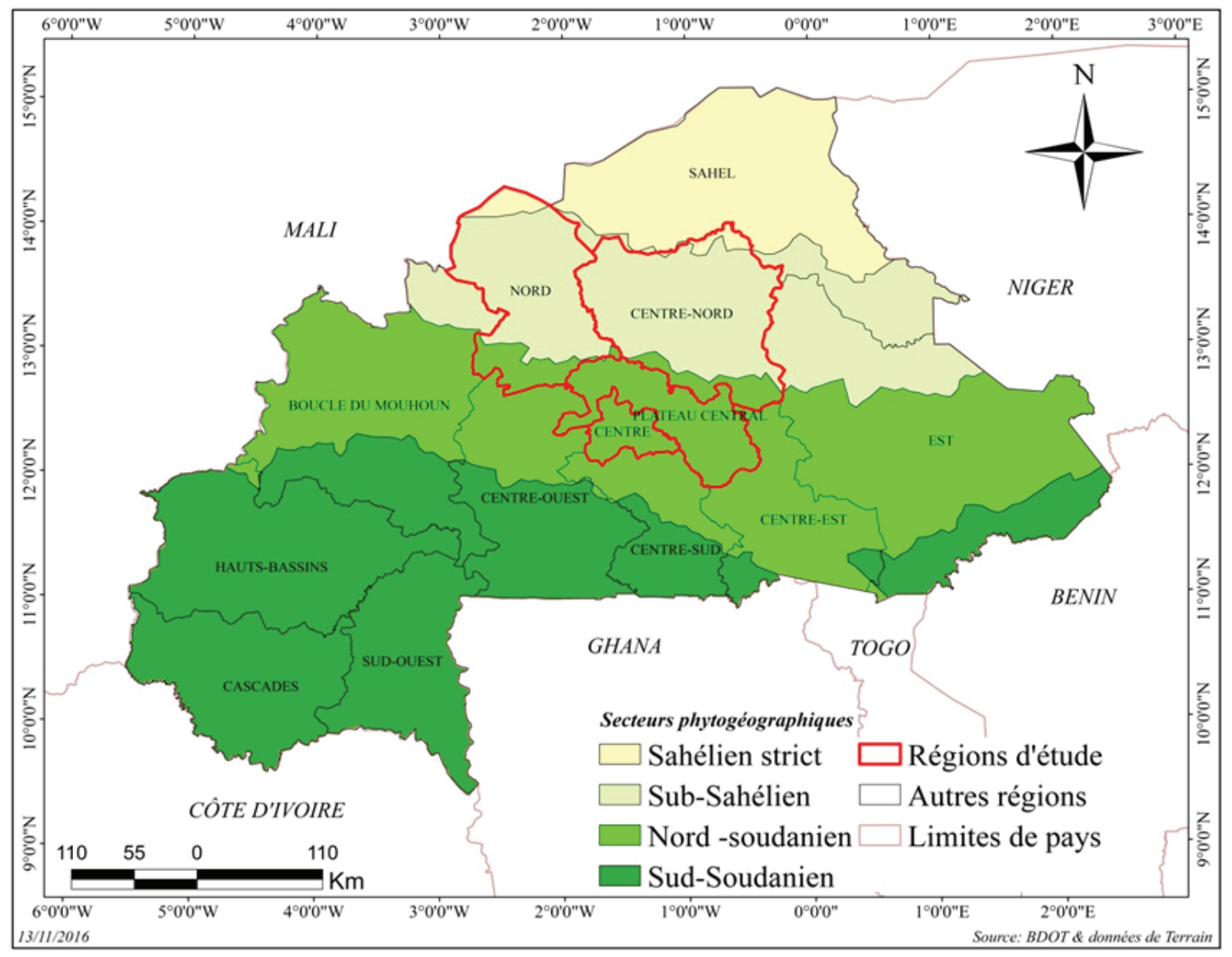

Fig. 1: Localisation de la zone d'étude / Location of the study area. 
le choix d'une seconde localité est désormais orienté par le même guide avec l'aide des autorités coutumières locales. Cependant, certaines localités voisines qui n'ont pas été recommandées par ces autorités ont été également prises en compte. Cela répond au souci d'obtenir des données représentatives et plus fiables, considérant les risques éventuels d'omission et d'ignorance de la part des autorités.

Les interviews ont concerné 1437 personnes autochtones. En effet, les autochtones sont plus habilités à fournir des informations fiables sur les critères de dénomination des espèces végétales relatives à l'ethnotaxonomie (SAVADogo et al. 2011; SAVAdogo 2013). Les entretiens se sont déroulés en langues locales Mooré, précisément dans les dialectes des différentes localités, avec l'aide d'interprètes. Le guide d'entretien a permis de recueillir les informations suivantes: les noms des différentes localités, les noms vernaculaires des espèces et leurs significations, des synonymes et des homonymes de ces noms vernaculaires, les critères de dénomination, le nombre d'espèces par critère de dénomination, ...

\subsection{Analyse des données}

Les plantes citées en langues locales sont identifiées par leurs noms scientifiques sur le terrain ou le cas échéant, des échantillons d'herbier ont été collectés pour être déterminés au laboratoire de l'Herbier de l'Université de Ouaga I Professeur Joseph Ki Zerbo. La nomenclature utilisée pour la détermination est celle de ArbonNier (2000) et ThiombIANO et al. (2012). Lorsque la plante citée n'existe plus dans les terroirs étudiés, son nom local est retenu pour être identifié avec l'appui des systématiciens qui parlent la langue.
Les données d'enquête ont été dépouillées puis saisies sur Excel. Les résultats ont été présentés sous forme de texte descriptif. Un tableau synthétique a été élaboré, faisant ressortir les noms des plantes en langue locale Mooré, leurs noms scientifiques, les critères utilisés dans la nomenclature et leurs désignations.

Des analyses statistiques (ANOVA) des données sur les effectifs des personnes interviewées ont été réalisées grâce au test de student, au seuil de 5\%., disponible sur JMP 8. Les effectifs ont été comparés deux à deux afin d'appréhender la significativité des différences entre ces effectifs. Les analyses floristiques ont été faites sur Excel. La composition floristique des espèces recensées a été présentée sous forme de tableau. Les spectres des principales familles ont été présentés sous forme de diagramme circulaire.

\section{RÉSULTATS}

\subsection{Répartition taxonomique des espèces citées par les enquêtés}

L'enquête ethnobotanique a permis de recenser au total 72 espèces végétales reparties en 51 genres et 29 familles. L'analyse des principales familles montre une dominance des Fabaceae-Mimosoideae $(11,1 \%)$ et des Commelinaceae $(11,1 \%)$ (Fig. 2). Elles sont suivies des Anacardiaceae $(8,33 \%)$, des Euphorbiaceae (6,9\%), des Poaceae $(6,9 \%)$, des Malvaceae $(5,6 \%)$ et des Fabaceae-Caesalpinioideae (5,6 \%). Les Apocynaceae, les Asteraceae, les FabaceaeFaboideae et les Lamiaceae sont peu représentées $(4,2$ chacune). Les autres familles représentent $27,8 \%$ de cette flore (Fig. 2).

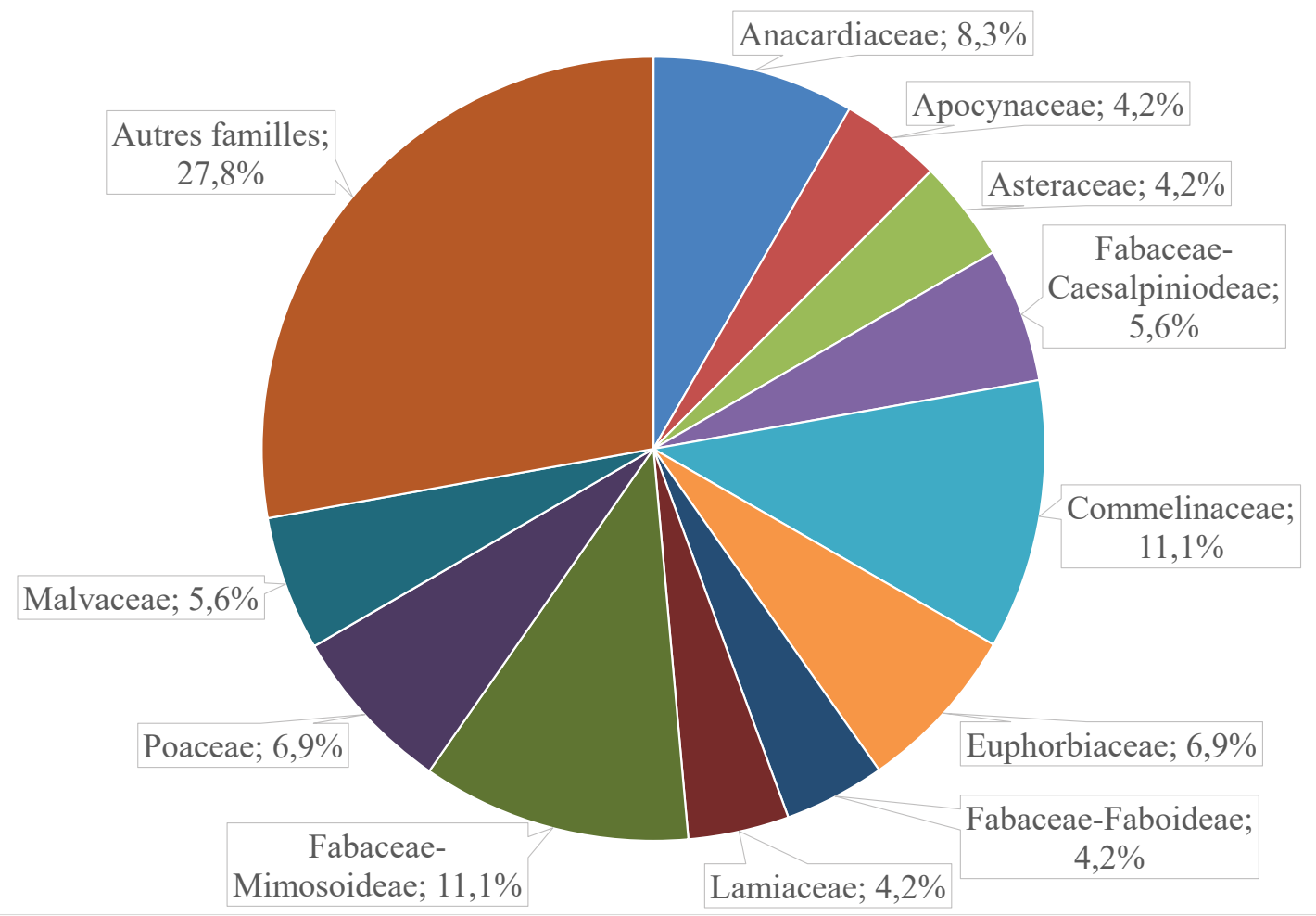

Fig. 2: Proportion centésimale des principales familles des espèces décrites par les Mossé / Centesimal proportion of the main families of the species described by Mossé. 


\subsection{Critères utilisés en ethnotaxonomie}

Les dénominations vernaculaires des espèces en langue mooré relèvent de plusieurs démarches. La distinction des espèces est faite quelque fois sur la base de caractères propres à chacune d'elles. Ces caractères concernent aussi bien l'usage que les caractéristiques morphologiques et écologiques. Les taxons sont également dénommés par des comparaisons. Les différentes démarches qui aboutissent à la dénomination et à l'identification des espèces végétales en pays Moagha ont été consignées dans des tableaux (Tableau 1 et 2). Le tableau 2 donne les détails sur l'étymologie et la signification littérale des dénominations vernaculaires des 72 espèces décrites par les Mossé. est beaucoup utilisée par la population locale pour les soins des nouveaux nés. La décoction (tisane) de ses feuilles est utilisée pour purger et laver les bébés.

Les tiges de Waltheria indica sont utilisées par les enfants pour la cueillette des fruits de Diospyros mespiliformis. Ainsi, l'espèce porte le nom de Gan raogo qui veut dire «bois pour transporter les fruits de Diospyros mespiliformis». Dans d'autres localités, elle porte également le nom de Yaryamdé (décoction ou tisane des Yarssé). Les Yarssé constituent une tribu de l'ethnie majoritaire du Burkina Faso, les Mossé. Ce peuple apprécie la décoction de Waltheria indica comme tisane des enfants, d'où son nom en référence à cette tribu.

Tableau 1: Pourcentages de citation des critères de dénomination des espèces par les Mossé / Table1: Percentage of citation of species naming criteria by Mossé

\begin{tabular}{|l|c|c|}
\hline Critères & $\begin{array}{l}\text { Pourcentage de } \\
\text { citation (\%) }\end{array}$ & $\begin{array}{l}\text { Nombre d'espèces } \\
\text { recensées (décrites) }\end{array}$ \\
\hline Usage fait de la plante & 93,59 & 10 \\
\hline Mysticisme de l'espèce & 85,9 & 4 \\
\hline Ecologie ou milieu de vie de l'espèce & 83,34 & 4 \\
\hline La dualité male/femelle & 82,6 & 7 \\
\hline Couleur des organes ou parties de la plante & 81,2 & 3 \\
\hline Origine de la plante & 79,8 & 4 \\
\hline Morphologie foliaire & 75,8 & 1 \\
\hline Présence d'organes saillants sur la plante & 75,29 & 4 \\
\hline Mode de dissémination des fruits ou des graines (la zoochorie) & 74,37 & 4 \\
\hline Morphologie des fruits & 62,7 & 2 \\
\hline Position des fruits sur la plante & 60,12 & \\
\hline Résistance au stress hydrique et au piétinement & 48,25 & 2 \\
\hline Couleur du latex ou de la sève & 44,9 & 10 \\
\hline Le gout ou l'odeur et la toxicité des organes & 43,23 & 3 \\
\hline Port de la plante & 42,71 & 3 \\
\hline Ressemblance de la plante à une autre espèce & 35,2 & 2 \\
\hline
\end{tabular}

Les analyses statistiques ne montrent aucune différence significative entre les effectifs des personnes interviewées par localité $(\mathrm{F}[3 ; 84]=1,83 ; \mathrm{p}=0,24)$.

Les critères les plus cités par la population (Tableau 1) sont l'usage fait de la plante $(93,6 \%)$, le mysticisme lié à l'espèce $(85,9 \%)$, l'écologie ou le milieu de vie de l'espèce $(83,3 \%)$, la dualité mâle/femelle $(82,6 \%)$, la couleur des organes ou parties de la plante $(81,2 \%)$, l'origine de la plante $(79,8 \%)$, la morphologie foliaire $(75,8 \%)$, la présence d'organes saillants sur la plante $(75,3 \%)$ et le mode de dissémination des fruits ou des graines $(74,4 \%)$.

\subsubsection{Usage fait de la plante}

Souvent, lorsqu'une espèce est utilisée, elle porte le nom de l'usage qui en est fait. Les exemples foisonnent dans ce domaine. Ce sont:

Cassia sieberiana qui porte le nom de Yamtiiga (arbre utilisé pour la préparation des tisanes pour bébés). Cette espèce
Caralluma adscendens et Desmidorchis acutangula portent toutes le nom Toubr-tiim (littéralement $=$ remède pour les maux d'oreilles) car selon la population, la sève de ces deux (02) espèces serait efficace contre les maux d'oreilles.

Ocimum basilicum et Ocimum americanum sont toutes des espèces dont la poudre des graines est utilisée pour débarrasser l'œil de ses impuretés et, de ce fait, les deux espèces portent chacune le nom de Nin-kansega (littéralement = nettoyeur de l'œil).

Bauhinia rufescens porte le nom vernaculaire Ti poega (littéralement $=$ arbre pour consultation ou pour la divination). Les rameaux de cette espèce seraient utilisés dans les pratiques divinatoires afin de prédire l'issue favorable ou non d'une action à entreprendre.

Ipomoea asarifolia est une espèce spontanée permettant de faire face au ruissellement de l'eau et à la dégradation des sols. C'est dans ce sens que la population l'a nommé Kogol- 
Tableau 2: Liste des espèces recensées indiquant leurs noms vernaculaire en langue Mooré, les critères de nomenclature et leurs significations littérales / Table 2: List of species inventoried with their vernacular names in Mooré language, nomenclature criteria and their literal meanings.

\begin{tabular}{|c|c|c|c|}
\hline Noms scientifiques & $\begin{array}{l}\text { Noms vernaculaires } \\
\text { (en langue mooré) }\end{array}$ & Significations des noms & Critères de dénomination \\
\hline Acacia dudgeoni & Gonga & Epine & $\begin{array}{l}\text { Présence d'un organe qui fait } \\
\text { sailli sur l'espèce }\end{array}$ \\
\hline Acacia erythrocalyx & Kango & Fourré & Le port de la plante \\
\hline Acacia gourmaensis & Gonga & Epine & $\begin{array}{l}\text { Présence d'un organe qui fait } \\
\text { sailli sur l'espèce }\end{array}$ \\
\hline Acacia senegal & Gon pèlga & Epine & $\begin{array}{l}\text { Présence d'un organe qui fait } \\
\text { sailli sur l'espèce }\end{array}$ \\
\hline Acacia seyal & Gon miiga & Epine & $\begin{array}{l}\text { Présence d'un organe qui fait } \\
\text { sailli sur l'espèce }\end{array}$ \\
\hline Acanthospermum hispidum & Guiéma taanbo & Trois tresses & $\begin{array}{l}\text { Présence de trois excroissances } \\
\text { sur le fruit }\end{array}$ \\
\hline Albizia chevalieri & Ronsindonga & Pseudo néré ou proche du néré & $\begin{array}{l}\text { Ressemblance de ses feuilles à } \\
\text { celles du néré }\end{array}$ \\
\hline Albuca nigritana & Zangsimdé ou Zagme yindga & $\begin{array}{l}\text { Même si elle est déterrée, son } \\
\text { flétrissement dure plusieurs } \\
\text { jours/Provoque des démangeai- } \\
\text { sons rectales }\end{array}$ & $\begin{array}{l}\text { Résistance au stress hydrique / } \\
\text { Effet des substances de l'espèce } \\
\text { sur l'organisme }\end{array}$ \\
\hline Ampelocissus leonensis & Liouli-sibi & Résinier des oiseaux & $\begin{array}{l}\text { Fruits très appréciés par les } \\
\text { oiseaux }\end{array}$ \\
\hline Anonna senegalensis & Bag-taama & Karité du chien & Fruits appréciés par les chiens \\
\hline Azadirachta indica & Nasar-sabga & Résinier du blanc & $\begin{array}{l}\text { Ressemblance des fruits à ceux } \\
\text { de Lannea microcarpa }\end{array}$ \\
\hline Bauhinia rufescens & Ti poega & $\begin{array}{l}\text { Plante utilisée pour des pra- } \\
\text { tiques divinatoires }\end{array}$ & Usage fait de l'espèce \\
\hline Biophytum umbraculum & Goudif naon timloogué & Plie tes pieds pour que je passe & Thigmotropisme de l'espèce \\
\hline Blumea aurita & Katré tabré & Le tabac de l'hyène & Ressemblance au pied de tabac \\
\hline Brachystelma bengeri & Liouli sengo & Brachystelma des oiseaux & $\begin{array}{l}\text { Espèce appréciée par les oi- } \\
\text { seaux }\end{array}$ \\
\hline Caralluma dalzielii & Toubr-tiim & $\begin{array}{l}\text { Médicament contre les maux } \\
\text { d'oreilles }\end{array}$ & Usage fait de la plante (La sève) \\
\hline Caralluma retrospisciens & Toubr-tiim & $\begin{array}{l}\text { Médicament contre les maux } \\
\text { d'oreilles }\end{array}$ & Usage fait de la plante (La sève) \\
\hline Cassia sieberiana & Yam tiiga ou Koumbri saka & $\begin{array}{l}\text { Arbre utilisé pour les tisanes } \\
\text { des bébés }\end{array}$ & $\begin{array}{l}\text { Usages fait de la plante (La } \\
\text { sève) }\end{array}$ \\
\hline Cenchrus biflorus & Yons-tabdo & Qui s'accroche aux animaux & Dissémination par zoochorie \\
\hline Commelina benghalensis & Boanga & Résiste aux socs comme un âne & $\begin{array}{l}\text { Résistance au piétinement et au } \\
\text { stress hydrique }\end{array}$ \\
\hline Commelina diffusa & Boanga & Résiste aux socs comme un âne & $\begin{array}{l}\text { Résistance au piétinement et au } \\
\text { stress hydrique }\end{array}$ \\
\hline Commelina africana & Boanga & Résiste aux socs comme un âne & $\begin{array}{l}\text { Résistance au piétinement et au } \\
\text { stress hydrique }\end{array}$ \\
\hline Commelina communis & Boanga & Résiste aux socs comme un âne & $\begin{array}{l}\text { Résistance au piétinement et au } \\
\text { stress hydrique }\end{array}$ \\
\hline Commelina forskalaei & Boanga & Résiste aux socs comme un âne & $\begin{array}{l}\text { Résistance au piétinement et au } \\
\text { stress hydrique }\end{array}$ \\
\hline Corchorus olitorius & Boulvang yanga & Corchorus femelle & Taille du limbe \\
\hline Corchorus tridens & Boulvang raaga & Corchorus mâle & Taille du limbe \\
\hline Crateva adansonii & Koulguin toega & Le baobab des mares & Ecologie de l'espèce \\
\hline Crotalaria pallida & Wind lebendé & $\begin{array}{l}\text { Qui s'oriente suivant la direc- } \\
\text { tion du soleil }\end{array}$ & $\begin{array}{l}\text { Orientation des faces supéri- } \\
\text { eures des feuilles suivant la } \\
\text { direction du soleil }\end{array}$ \\
\hline Cyanotis arachnoidea & Ting-kui tim-kui & $\begin{array}{l}\text { Il faut que la terre soit sèche } \\
\text { pour que je périsse }\end{array}$ & Résistance au stress hydrique \\
\hline
\end{tabular}




\begin{tabular}{|c|c|c|c|}
\hline Noms scientifiques & $\begin{array}{l}\text { Noms vernaculaires } \\
\text { (en langue mooré) }\end{array}$ & Significations des noms & Critères de dénomination \\
\hline Cyanotis lanata & Ting-kui tim-kui & $\begin{array}{l}\text { Il faut que la terre soit sèche } \\
\text { pour que je périsse }\end{array}$ & Résistance au stress hydrique \\
\hline Cyanotis longifolia & Ting-kui tim-kui & $\begin{array}{l}\text { Il faut que la terre soit sèche } \\
\text { pour que je périsse }\end{array}$ & Résistance au stress hydrique \\
\hline Delonix regia & Nasar ronga & $\begin{array}{l}\text { Néré de l'homme à la peau } \\
\text { blanche }\end{array}$ & Ressemblance au Néré \\
\hline Dialium guineense & Mak poussa & Tamarin de la Mecque & Origine de l'espèce \\
\hline Digitaria horizontalis & Tin-tim-tin & Etalement au sol de l'espèce & La biologie de l'espèce \\
\hline Eleusine indica & Targanga & Solide, résistante & Résistance au piétinement \\
\hline Eragrostis tenella & Liouli saaga & Balaie des oiseaux & Ressemblance au balai \\
\hline Euphorbia convolvuloides & Bouli bisoum & Lait du Chevreau & $\begin{array}{l}\text { Présence de latex blanc lai- } \\
\text { teux }\end{array}$ \\
\hline Euphorbia heterophylla & Bouli bisoum & Lait du Chevreau & $\begin{array}{l}\text { Présence de latex blanc lai- } \\
\text { teux }\end{array}$ \\
\hline Euphorbia hirta & Bouli bisoum & Lait du Chevreau & $\begin{array}{l}\text { Présence de latex blanc lai- } \\
\text { teux }\end{array}$ \\
\hline Ficus sur. & Womseiga & $\begin{array}{l}\text { Répartition des fruits jusqu'au } \\
\text { tronc }\end{array}$ & Position des fruits sur l'arbre \\
\hline Gardenia sokotensis & Tang rakoenga & Bois sec de la colline & Ecologie et l'aspect du bois \\
\hline Hibiscus sabdariffa & Weguêd miougou & Hibiscus rouge & Couleur des calices de la fleur \\
\hline Hiptis spicigera & Youni younga & Herbe odorante & Odeur de la plante \\
\hline Ipomoea aquatica & Koul bengdo & Herbe des mares & Ecologie de l'espèce \\
\hline Ipomoea asarifolia & Kogol koom ou Kokoka & $\begin{array}{l}\text { Protection contre le ruisselle- } \\
\text { ment de l'eau }\end{array}$ & Usage fait de la plante \\
\hline Jatropha curcas & Wonbim-Bangma. & $\begin{array}{l}\text { Goûte mois tu sauras qui je } \\
\text { suis }\end{array}$ & $\begin{array}{l}\text { Effet des substances de } \\
\text { l'espèce sur l'organisme }\end{array}$ \\
\hline Lannea acida & Sab toulga & $\begin{array}{l}\text { Lannea servant à confection- } \\
\text { ner des pilons }\end{array}$ & $\begin{array}{l}\text { Usage fait de la plante (le bois } \\
\text { utilisé pour la confection des } \\
\text { mortiers) }\end{array}$ \\
\hline Lannea kerstingii & Waam sabga & Lannea du singe & $\begin{array}{l}\text { L'animal qui apprécie les } \\
\text { fruits de l'espèce }\end{array}$ \\
\hline Lannea microcapa & Sab boega & $\begin{array}{l}\text { Lannea dont l'odeur des fruits } \\
\text { est comparée à celle du bouc }\end{array}$ & Odeur des fruits \\
\hline Lannea velutina & Waam sabga & Lannea du singe & $\begin{array}{l}\text { L'animal qui apprécie les } \\
\text { fruits de l'espèce }\end{array}$ \\
\hline Moringa oleifera & Argin tiiga & Arbre du paradis & Origine de l'espèce \\
\hline Nelsonia canescens & Koulg poussa & Tamarin des zones humides & Ecologie de l'espèce \\
\hline Ocimum americanum & Nin kaasga & Nettoyeur de l'œil & Usage fait de la plante \\
\hline Ocimum basilicum & Nin kaasga & Nettoyeur de l'œil & Usage fait de la plante \\
\hline Ozoroa insignis & Neb noyan & $\begin{array}{l}\text { Les maléfices ou les mauvais } \\
\text { sorts proférés par les humains }\end{array}$ & $\begin{array}{l}\text { Le caractère mystique dont } \\
\text { incarne l'espèce }\end{array}$ \\
\hline Parkia biglobosa & Roanga & Couleur jaune de la pulpe & Couleur de la pulpe des fruits \\
\hline Paulinia pinnata & Noussa nou & Cinq doigts & Forme digiti-lobée des feuilles \\
\hline Phyllanthus amarus & $\begin{array}{l}\text { Wom pooré ou Roguin } \\
\text { pooré }\end{array}$ & $\begin{array}{l}\text { Fructification sur le dos } \\
\text { (feuilles) }\end{array}$ & $\begin{array}{l}\text { Position des fruits sur la } \\
\text { plante }\end{array}$ \\
\hline Piliostigma reticulatum & Baguin daaga & Piliostigma mâle & Taille du limbe \\
\hline Piliostigma thonningii & Baguin yanga & Piliostigma femelle & Taille du limbe \\
\hline Sclerocarya birrea & Nob raogo & Sclerocarya mâle & Absence de productivité \\
\hline Sorghum bicolor & Ka zinga & Sorgho rouge & $\begin{array}{l}\text { Couleur des glumes ou des } \\
\text { graines }\end{array}$ \\
\hline Stereospermum kunthianum & Widig zaka ou Nin yilinga & $\begin{array}{l}\text { Détruit la famille ou provoque } \\
\text { des vertiges }\end{array}$ & Fumée hallucinogène du bois \\
\hline Trianthema portilastrum & Kour-kour yamdo & Herbes ou fourrage du porc & Usage faite de la plante \\
\hline
\end{tabular}




\begin{tabular}{|l|l|l|l|}
\hline Noms scientifiques & $\begin{array}{l}\text { Noms vernaculaires } \\
\text { (en langue mooré) }\end{array}$ & Significations des noms & Critères de dénomination \\
\hline Trichilia emetica & Kinkirs taanga & Le karité des esprits & Ressemblance au karité \\
\hline Triumfetta pentandra & Natoun- kouli & Je te suis pour aller chez toi & $\begin{array}{l}\text { Présence des crochets ou des } \\
\text { appendices sur les fruits (dis- } \\
\text { sémination par zoochorie) }\end{array}$ \\
\hline Triumfetta rhomboidea & Natoun- kouli & Je te suis pour aller chez toi & $\begin{array}{l}\text { Présence des crochets ou des } \\
\text { appendices sur les fruits }\end{array}$ \\
\hline Vitex crysocarpa & And raaga & Vitex mâle & Dualité mâle/femelle \\
\hline Voacanga africana & Boega lanyiiba & Testicule bilobé du bouc & Forme du fruit \\
\hline Waltheria indica & Gan raogo & $\begin{array}{l}\text { Support pour les fruits de Di- } \\
\text { ospyros mespiliformis }\end{array}$ & Utilité du rameau de la plante \\
\hline Ziziphus mucronata & Mouguin toaga & Zizuphus amer & Le goût des fruits \\
\hline Zornia glochidiata & Peseg yamdo & Herbe du mouton & Usage fait de la plante \\
\hline
\end{tabular}

koom ou Kokoka (gardien de l'eau ou barrière contre le ruissellement de l'eau).

Le bois de Lannea acida est beaucoup utilisé dans la confection des pilons. Ce qui justifie son appellation Sabtoulga qui signifie, Lannea servant à la confection de pilon.

Trianthema portulacastrum est une herbe très appréciée des porcs. Pour faire allusion au fourrage des porcs, les populations l'ont nommé kourkouri-yamdo (littéralement = herbe du porc).

\subsubsection{Caractère mystique de l'espèce}

Les organes de Ozoroa insignis (rameau, écorce,...) fixés dans certains lieux bien précis (champs de cultures, habitat, ...) permettent de conjurer les mauvais sorts pour certaines personnes. Ce caractère mystique reconnu à l'espèce lui vaut son appellation Neb-noyan qui signifie «paroles des gens».

Le bois de Stereospermum kunthianum transporté dans une concession est source de malheurs aux dires des enquêtés. C'est ce qui explique bien son appellation «Widig-zaka» (destructeur de famille) ou «Ni-yilinga» (vertige, hallucination) ou encore «Yiiga» (vision flou). C'est pourquoi chez les Mossé, le bois de l'espèce n'est pas transporté à la maison, à plus forte raison utilisé comme bois de chauffe. L'espèce Crotalaria pallida porte les noms Wind-lebendé (littéralement $=$ qui est dirigé vers le soleil) par ce que ses feuilles s'orientent toujours en direction du soleil; et $\mathrm{Na}$ maag-beogo (littéralement $=$ le malade va guérir demain) car l'espèce est hautement médicinale.

Le thigmotropisme chez l'espèce Biophytum umbraculum lui confère une propriété spectaculaire selon les mossé. En effet, elle pli ses feuilles quand on la touche. La population l'appelle à cet effet, goudif-naw-tim-loogué (littéralement = pli tes pieds pour que je passe).

\subsubsection{L'écologie ou le milieu de vie de l'espèce}

Suivant le milieu écologique, on peut avoir plusieurs appellations. Les espèces telles Nelsonia canescens, Crateva adansonii, Ipomoea aquatica et Gardenia sokotensis sont des exemples illustratifs. Ainsi, toutes les espèces qui colonisent les milieux humides, aquatiques, inondables ou les bas-fonds portent le préfixe mooré Koulg ou Koulga qui évoque la présence de l'eau suivi du radical du nom auquel l'espèce présente une très forte ressemblance. Quelques noms vernaculaires en langue mooré et leurs significations citées par les populations sont:

- Koulg-poussa qui signifie Tamarin de la mare (Nelsonia canescens) ou Ting- poussa (Tamarin rampant)

- Koulg-bengdo qui signifie haricot ou feuille de la mare (Ipomoea aquatica)

- Koulg-toega ou Kalguin-toega qui veut dire baobab de la mare (Crateva adansonii)

- Quant aux espèces des collines et toute autre élévation semblable, elles portent le préfixe Tang ou Tanga en référence à la colline. C'est le cas de Gardenia sokotensis qui colonise le plus souvent les collines et porte de ce fait le nom de Tang-rakoenga (littéralement bois sec de colline).

\subsubsection{La dualité mâle femelle ou au sexe de la plante}

Les dénominations utilisées dans ce cas sont les préfixes Raogo ou Raaga qui signifient mâle et Yaanga qui veut dire femelle. C'est ainsi que Corchorus tridens porte le nom de Boulvang raaga (Corchorus mâle) et Corchorus olitorius Boulvang yanga (Corchorus femelle).

Il y a aussi l'exemple de Piliostigma reticulatum dont le nom «Bangin raaga» signifie Piliostigma mâle et celui de Piliostigma thonningii «Bangin yanga» (Piliostigma femelle).

Ces dénominations sont également utilisées sur d'autres espèces pour exprimer leur caractère dioïque. Ce sont Sclerocarya birrea (Nob-raaga ou Nob-yaanga), Vitex crysocarpa (And-raaga ou And-yaanga) et Lannea microcarpa (Sabraaga ou Sab-yaanga)...

\subsubsection{La couleur des organes ou parties de la plante}

Hibiscus sabdariffa à fleur rouge et dont les calices sont utilisés pour préparer le bissap porte le nom de «Weguêdmiiga» ou «Weguêd-miougou» qui veut dire littéralement $H$. sabdariffa rouge. Cette espèce doit son nom à la couleur rouge de ses calices. Il y a aussi le cas de Parkia biglobosa. Elle porte un nom qui reflète la couleur «jaune» de la pulpe de ses fruits à maturité. Ainsi, l'arbre porte le nom «Roan- 
ga» et ses fruits portent le nom «Rondo» (littéralement $=$ couleur jaune).

La couleur des glumes et celles des graines de certains Poaceae peuvent être utilisées dans la dénomination des espèces en Mooré. Les exemples les plus courants sont:

- $\quad$ Ka zinga (littéralement $=$ sorgho rouge) pour désigner le Sorghum bicolor à graines rouges.

- Fib miougou (sorgho à glumes rouges) pour désigner le Sorghum bicolor à glumes rouges.

\subsubsection{L'origine de la plante}

La plupart des espèces exotiques introduites au Burkina Faso portent des noms qui reflètent leur origine. En général, ces noms sont précédés d'un préfixe qui indique l'origine de la plante, le radical étant celui d'une plante locale avec qui la ressemblance est établie. Les préfixes les plus courants sont: «Mak», «Argin», «Nasar»,...

«Mak» signifie Mecque pendant que «Argin» signifie paradis. Ainsi, toute espèce qui porte l'un de ces préfixes serait originaire de la Mecque. Le préfixe «Nasar» veut dire l'homme à la peau blanche (l'Homme blanc). Une espèce qui porte ce préfixe proviendrait de l'Occident. Cependant, il convient de mentionner qu'aucun d'entre eux ne provient effectivement de l'Europe, bien que les Français puissent les avoir amenés et / ou encouragé leur culture.

Ces dénominations sont portées par des espèces telles que:

- Dialium guineense; appelée Mak-poussa qui signifie littéralement «Tamarin de la Mecque».

- Moringa oleifera est appelée Argin-tiiga. Celui-ci peut être décomposé en Argin $=$ paradis et Tiiga $=$ arbre. Ainsi Argin-tiiga signifie littéralement «arbre du paradis». Selon la population, cette espèce est originaire de la Mecque, ville sainte de l'Islam alors comparée au paradis.

- Azadirachta indica est appelée Nasar-sabga. Ce nom signifie "vigne du blanc "pour traduire la ressemblance de ses fruits aux raisins.

- Delonix regia est appelée Nasar-roanga ce qui traduit l'expression "néré du blanc" car les Mossé estiment qu'elle ressemble plus au néré.

\subsubsection{Morphologie foliaire}

La morphologie foliaire est l'un des critères couramment utilisés par les Mossé pour nommer les espèces. C'est l'exemple de Paullinia pinnata dont le nom vernaculaire en langue mooré est Nousa-nou. Littéralement ceci veut dire cinq (05) doigts. Le nom mooré de cette espèce fait référence à ses feuilles digitées, rappelant les cinq doigts de la main humaine.

\subsubsection{La présence de certains organes saillants sur la plante}

Toutes les espèces d'Acacia portent le même nom en Mooré, celui de Goanga. Dans cette appellation, allusion est faite aux épines des Acacia car, Goanga signifie épine. Cependant, selon la couleur du tronc de l'arbre ou du caractère coriace de l'épine, des précisions peuvent subsister. Par exemple, Acacia seyal dont le tronc peut prendre une couleur légèrement rougeâtre porte le nom de Gong-miiga (littéralement= épine rouge) pour faire allusion à la couleur du tronc. Acacia senegal porte le nom de Gong-pelga (littéralement = épine blanc) pour faire allusion à la couleur blanchâtre de son tronc. Il y a Gong-sablega (littéralement = épine noire) pour faire allusion à Acacia dudgeoni. Quant au caractère coriace de l'épine, on peut citer Gong-payandga (littéralement $=$ épine coriace), non vernaculaire de Acacia gourmaensis.

\subsubsection{Mode de dissémination des fruits ou des graines (la zoochorie)}

Les Mossé se réfèrent également aux organes de dissémination des espèces pour leurs dénominations. Ainsi, les graines des espèces telles Triumfetta rhomboidea, T. pentandra, Zornia glochidiata toutes pourvues d'appendices et/ou de crochets, pouvant s'accrocher aux poils des animaux ou aux vêtements des hommes et transportées par eux jusqu'à leur destination sont appelées Na-toun-kouli qui veut dire littéralement «je te suis jusqu'à ton domicile». Cenchrus biflorus porte le nom de Yons-tabdo (littéralement = qui s'accroche). Ce nom est donné à cette espèce pour faire allusion à la capacité de son épi à s'accrocher aux animaux et aux hommes qui assurent sa dissémination (la zoochorie).

\subsubsection{Morphologie des fruits}

L'espèce la plus connue, obéissant à ce critère de nomenclature est Voacanga africana. En langue mooré, le nom de cette espèce est Boeg-laanyiiba (littéralement = testicules bilobés du bouc), pour faire allusion aux fruits bilobés de l'espèce. Il y a également Acanthospermum hispidum qui porte le nom de Guièma tâabo qui signifie «trois tresses ou trois épines». Dans cette dénomination, allusion est faite aux trois épines portées par le fruit de l'espèce.

\subsubsection{La position des fruits sur la plante}

Cette position occupe une place importante dans les dénominations des plantes en langue mooré. On peut citer le cas de Ficus sur dont les fruits s'étendent jusqu'au tronc (caulicarpie). De ce fait, l'espèce est appelée Wom-seiga qui signifie fructification à la «hanche» ou sur le tronc. Il y a également le cas de Phyllanthus amarus qui forme ses fruits sur la face inférieure des feuilles. Pour cette raison, elle est nommée par les Mossé Wom-pooré (fructification sur le dos) ou roguin-pooré (mettre bas sur le dos).

\subsubsection{La résistance au stress hydrique et /ou au piétine- ment}

Plusieurs exemples d'espèces peuvent être cités dans ce cas. Ce sont:

- les espèces des genres Commelina (Commelina benghalensis, C. diffusa, C. africana, C. communis, C. forskalaei, ...) et Cyanotis (C. lanata, C. arachnoidea, C. longifolia ...). A cause de leur appareil végétatif très charnu, ces espèces résistent longtemps au stress hydrique et à la dessiccation. Ainsi, elles portent la même 
dénomination Tingkui- timkui (littéralement = pour que je dessèche, il faut que la terre soit sèche) ou Bonga (littéralement $=$ âne) pour faire allusion à la forte résistance de l'âne aux travaux.

- Albuca nigritana, grâce à son bulbe et à ses feuilles très charnues, présente une forte résistance au stress hydrique. Et, même si on la déterre, elle peut vivre pendant plusieurs jours. Toutes ces qualités lui ont valu l'appellation de Zangsindé (literalement = qui refuse de mourir).

Eleusine indica porte le nom de Targanga (littéralement $=$ résistant ou solide) car l'espèce présente une certaine solidité et résiste très bien au piétinement des hommes et des animaux.

\subsubsection{La couleur du latex de l'espèce}

Le couleur du latex de certaines plantes, en l'occurrence la couleur blanche est comparée au lait et est souvent utilisée dans la dénomination des espèces végétales. Ainsi, la plupart des espèces du genre Euphorbia (E. hirta, E. heterophylla, E. convolvuloides, ...) portent toutes le même nom Bouli-bissoum en langue vernaculaire mooré, qui signifie «lait des chevreaux». L'usage du nom chevreaux vient du fait que ce sont des espèces très appréciées des chevreaux. Bouli-bissoum est donc une appellation métaphorique du latex blanc des espèces herbacées du genre Euphorbia que l'on compare au lait de chèvre.

\subsubsection{Le goût ou l'odeur des organes de la plante}

En langue mooré, lorsque l'organe d'une plante (fruit, racine, feuille, graine, ...) a un goût amer, le nom de la plante porte souvent le préfixe Toogo ou Toaga (littéralement = amer). C'est le cas de Ziziphus mucronata qui porte des fruits amers, qui ne sont pas consommés par la population; et que l'on appelle Mouguin toaga pour dire Ziziphus amer. Il y a aussi l'exemple de Jatropha curcas. Cette espèce doit son nom vernaculaire Wonbem-bangma à sa toxicité et à la répugnance que les animaux en ont. Littéralement, ce nom signifie «goûte moi et tu sauras qui je suis».

Hiptis spicigera, à cause de ses odeurs, prend le nom de youni younga qui signifie (beaucoup d'odeur).

\subsubsection{Le port de la plante}

Le port de la plante est un critère très souvent utilisé pour nommer les espèces lianescentes (volubiles, grimpantes, rampantes). L'appellation Kango qui signifie littéralement fourré, est donnée à l'espèce Acacia erythrocalyx pour faire allusion à son caractère lianescent faisant de son couvert une certaine densité impénétrable. Digitaria horizontalis quant à elle, est une mauvaise herbe, envahissante, rampante, pouvant coloniser une vaste superficie. C'est son caractère rampant qui lui vaut l'appellation «Tin-tim-tin» qui signifie «qui s'étale sur une grande surface».

\subsubsection{La ressemblance de l'espèce à une autre espèce ou sa consommation par d'autres espèces animales}

Pour ce critère de dénomination, l'exemple de Blumea aurita est très illustratif. Cette espèce porte le nom de Katre- tabré (tabac de l'hyène). Cette appellation vient du fait que Blumea aurita a une très forte ressemblance avec l'espèce Nicotiana tabacum (tabac). L'espèce Trichilia emetica qui présente une ressemblance avec le karité porte ainsi le nom de Kinkirs-tâanga (karité des esprits).

On peut citer d'autres exemples tels:

Baa-taama $($ Karité du Chien $)=$ Annona senegalensis car les chiens apprécient les fruits de cette espèce;

Waam-sabga $($ vigne du singe $)=$ Lannea velutina et Lannea kerstingii car les fruits de ces espèces ressemblent au raisin;

Albizia chevalieri porte le nom de Ronsin-donga (pseudodonga $=$ pseudo néré) car elle ressemble beaucoup au néré;

Eragrostis tenella (Liouli saaga, littéralement = balais des oiseaux) pour sa ressemblance au balai de petite taille (pour oiseaux);

Brachystelma bingeri (Liouli sinnega, littéralement $=$ Brachystelma des oiseaux) car ses tubercules sont appréciés par les oiseaux;

Ampelocissus leonensis (Liouli sibi $=$ raisin des oiseaux) pour sa ressemblance aux raisins et sa consommation par certains oiseaux.

\subsection{Variation des noms vernaculaires suivants les locali- tés: les synonymies et les homonymies}

Comme les dialectes, les noms vernaculaires en langue Mooré varient suivant les localités. Dans toute la région du nord du Burkina Faso, Cassia sieberiana et les espèces du genre Euphorbia s'appellent respectivement Koumbrisaka et Bouli bissoum tandis que dans la région du centre nord, elles prennent respectivement le nom de Yaamtiiga et walma missoum. Dans les provinces du Yatenga, du Zondoma et de Lorum, Stereospermum kunthianum porte le nom de Widigzaka alors que les populations de la province du Sanmentenga l'appellent Ni-yilinga et ceux de la province de Ganzourgou, Yiiga. L'appellation Zaguêm yindga ou Zaguêm sèega donnée à Albuca nigritana est spécifique à la région du nord, plus précisément dans la province du Yatenga. Dans le Plateau Central Mossé et dans la région du centre, son nom est Poanga ou zangsindè. Deux (02) autres espèces dont les noms varient aussi selon les régions sont Hyptis specigera et Ocimum americanum. L'une de ces deux espèces porte le nom de l'autre en fonction des localités. Dans toute la région du nord et certaines localités du Plateau Central, Hyptis spicigera porte le nom de Youni-yougga et $O$. americanum, Ni-kaasga. Ocimum basilicum porte également le nom Ni-kaasga dans toute la région du nord. Par contre, dans d'autres villages du Plateau Central et dans toute la région du centre, c'est carrément le contraire. Roung-roungui est attribué à Hyptis spicigera. Azadirachta indica, une espèce exotique, importée de l'Inde, garde son appellation en langue française Neem dans tout le territoire Moagha; cependant, cette espèce a une autre appellation, Nassar sabga dans beaucoup de villages des Mossé. Par ailleurs, il existe des homonymies dans la taxonomie des Mossé. En effet, toutes les espèces du genre Euphorbia (Euphorbia hirta, E. heterophylla, E. convolvuloides,...) s'appellent Bouli-Bissoum dans la région du nord et Wal-Bissoum dans la régi- 
on du centre nord, du Plateau Central Mossé et du centre. Dans l'appellation bouli-Bissoum (littéralement = lait du chevreau) ou Wal-Bissoum (littéralement = lait de la tourterelle), allusion est faite à la couleur et à la matière même du latex de ces espèces du genre Euphorbia qui ressemble à du lait. Les espèces du genre Ocimum (O. americanum, O. basilicum) portent chacune le nom de Nin-kansséga (littéralement $=$ nettoyeur de l'œil). Ce sont des homonymes. Ainsi, la poudre des graines de ces espèces serait un puissant nettoyeur de l'œil capable de débarrasser de celui-ci ses impuretés. Outre ces espèces on peut citer d'autres homonymes tels que les genres Zornia, Caralluma et Triumfetta. Ainsi, pour les paysans, Zornia glochidiata, T. rhomboidea et $T$. pentandra porte le même nom vernaculaire Na-tounkouli (littéralement $=$ je m'accroche à toi pour aller jusqu'à ton domicile). Caralluma adscendens et Desmidorchis acutangula portent toutes le nom Toubr-tiim (littéralement $=$ remède pour les maux d'oreilles).

\subsection{Perception paysanne et conservation des espèces}

La perception qu'ont les populations de certaines espèces épargne ces dernières de la pression humaine. Calotropis procera par exemple est une plante qui pousse abondamment dans les cimetières. Cet habitat lui confère un certain mysticisme et fait d'elle une espèce crainte des populations de certaines localités en pays Moagha.

Ozoroa insignis est également frappée de mysticisme car son bois sert à repousser les mauvais sorts. Ce qui le préserve contre les coupes. Selon les personnes interrogées, son écorce implantée dans un champ de mil ou de maïs par exemple, préserverait ces champs de toutes les mauvaises intentions.

Quant à Stereospermum kunthianum, elle est préservée des coupes du fait que son bois est considéré comme destructeur de familles pour certains Mossé. Selon certaines personnes, la fumée issue de la combustion du bois de cette espèce provoque des hallucinations. Pour d'autres, les feuilles de l'espèce seraient également susceptibles de provoquer des hallucinations d'où son appellation Niyilinga (vertige). C'est également pour cette même raison que plusieurs personnes défendent aux membres de leurs familles de s'assoir à l'ombre de Stereospermum kunthianum.

La place de certaines plantes dans la conception Moagha trouve son origine dans leurs spécificités phénologiques. C'est le cas de Faidherbia albida, qui à cause de l'inversion de son cycle de feuillaison par rapport aux autres espèces est largement réputée auprès de la population. Ainsi au nord du Burkina Faso, la perception populaire reconnaît en cette espèce une plante qui pourrait se passer de l'eau de pluie pour vivre dans la mesure où elle perd ses feuilles en saison des pluies et les retrouve en saison sèche.

Il existe également un mythe sur Maytenus senegalensis. Celui-ci réside dans le fait qu'elle est utilisée dans la fabrication de médicaments traditionnels pour stimuler la fertilité masculine et augmenter les chances de concevoir un enfant lors d'un rapport sexuel. Selon la population, l'utilisation de ce produit par un homme quelques minutes avant un rapport sexuel entraîne obligatoirement une grossesse; d'où l'appellation de tok-vougri qui signifie littéralement «une seule éjaculation».

Selon la population, Biophytum umbraculum est une espèce mystique en ce sens qu'en touchant ses feuilles et en lui demandant de les plier, les feuilles se plient. Elle ne sait pas que le thigmotropisme est un phénomène physiologique normal chez l'espèce Biophytum umbraculum et que sans même dire un mot, le simple toucher peut provoquer les plies des feuilles.

Adansonia digitata, Khaya senegalensis, Tamarindus indica, Afzelia africana, Ceiba pentandra, Gardenia ternifolia, Ficus sycomorus, Ficus ingens, Ficus thonningii, Ficus platyphylla, sont des espèces perçues comme des arbres qui incarneraient ou qui abriteraient des génies. Elles sont toutes craintes par les populations et sont dans certaines localités à l'abri d'exploitations multiformes humaines.

\section{Discussions}

\subsection{Logique et complexité de la nomenclature locale}

Les noms attribués à certaines espèces végétales paraissent complexe. Ainsi, selon les personnes interrogées, certaines plantes comme Piliostigma reticulatum, Piliostigma thonningii, Corchorus tridens, Corchorus olitorius, Terminalia macroptera, Terminalia avicennioides, présenteraient des pieds mâles et des pieds femelles. Dans ces cas, ce n'est pas la présence ou l'absence d'organes reproducteurs qui est mise en évidence mais plutôt les tailles des feuilles. Par exemple, Piliostigma reticulatum à cause de la taille du limbe de ses feuilles relativement plus petite que celle du limbe des feuilles de $P$. thonningii est appelée Piliostigma mâle (Banguin-daaga) tandis que $P$. thonningii est dénommée Piliostigma femelle (Banguin-yaanga). Selon ces mêmes critères, Corchorus tridens porte le nom de Corchorus mâle (Boulvang-raaga) et C. olitorius le pied femelle (Boulvangyaanga).

Pour le genre Terminalia, l'espèce Terminalia avicennioides dont les feuilles sont petites portent le nom de Kondré (Terminalia) tout simplement ou de Kond-raaga (Terminalia mâle). En revanche, T. macroptera dont les feuilles sont plus grandes porte le nom de Kond-poko (Terminalia femelle). Tous ces cas reflètent le caractère complexe de l'ethnotaxonomie dont les critères ne sauraient expliquer le fondement de toutes les dénominations.

Néanmoins, certains critères sont logiques et compatibles au sens donnés aux ethno taxons même si les auteurs de ces taxons peinent à fournir des argumentaires convaincants et cohérents. Ainsi, chez les Mossé, lorsqu'un pied est improductif, il prend le nom de pied mâle. Le mâle est toujours attribué aux pieds stériles. C'est dans ce sens que le pied improductif de Sclerocarya birrea est appelé Nob-raaga (Sclerocarya mâle), celui de Lannea microcarpa, Sab-raaga (Lannea mâle).

Les espèces dont les fruits sont odorants et dégageant une odeur fétide sont qualifiées d'espèces «Bouc» pour faire allusion à leur odeur. Ainsi, les variétés de Lannea microcarpa dont les fruits sont odorants prennent le nom de (Sab-boega) 
qui signifie Lannea «Bouc». Cela montre que les paysans ne se contentent pas seulement de données matérielles pour les dénominations des espèces mais prennent aussi en compte certaines valeurs intrinsèques immatérielles des plantes.

\subsection{Similarité entre critères taxonomiques des Mossé et ceux d'autres ethnies de l'Afrique}

L'ethnotaxonomie ou para taxonomie (SPICHIGER et al. 2000), dont le but est l'étude des systèmes de classification utilisés par les populations indigènes, constitue un moyen permettant de comprendre le mode de perception des plantes. Des informations intéressantes peuvent émaner de la signification littérale et de l'étymologie des dénominations vernaculaires.

Les démarches identifiées ci-dessus pour la dénomination des plantes par les Mossé du Burkina Faso s'observent également chez les Malinké de Côte d'Ivoire et d'autres groupes ethniques, mais toutefois avec quelques différences. Chez les Malinké, les espèces indigènes sont généralement dénommées par rapport aux caractères botaniques de l'organe utilisé, combiné ou non à l'usage (AMBÉ \& MALAISSE 2000).

Les références aux caractéristiques de la plante s'observent chez les Bambara du Mali et les Peulh du Cameroun. Dans ce dernier groupe ethnique, l'espèce Cassia hirsuta est nommée "Kaccu-Kaccunga", "sale chose puante", à cause du goût peu agréable du décocté des feuilles (AMBÉ \& MALAISSE 2000). De même, l'appellation "nyamm-jeeda" (mange / tais-toi) désigne un ensemble de plantes à sauce peu agréables à manger à cause de leur odeur. On doit pourtant s'en contenter quand on a faim. Il s'agit de Corchorus sp. (Tiliaceae), de Sida sp. (Malvaceae) et d'Urena lobata (Malvaceae).

Parmi les références aux caractéristiques morphologiques, on peut citer, pour le Mali, le cas du "sinjan" Cassia sieberiana et du "sinjam- ba" (grand sinjan) Kigelia africana. Ces deux espèces possèdent des fruits impressionnants par leur taille qui peut atteindre $80 \mathrm{~cm}$ de long pour $C$. sieberiana (Aubreville 1936) et 30 à $60 \mathrm{~cm}$ de long pour K. africana (Berhaut 1967). Cependant, les gousses de C. sieberiana sont étroites tandis que les fruits du saucissonnier Kigelia africana sont volumineux, atteignant 6 à $8 \mathrm{~cm}$ de large (BERHAUT 1967), ce qui vaut à cette dernière l'appellation de "grand sinjan".

En ce qui concerne le qualificatif de mâle ou femelle, il s'applique dans des cas divers, souvent pittoresques, parfois étonnants selon les populations considérées. Par exemple, les Mossé considèrent Piliostigma reticulatum (Banguin daaga) comme mâle et $P$. thonningii (Banguin yanga) comme femelle. Ce qui n'est pas le cas chez les Peulh de Côte d'Ivoire pour qui Piliostigma reticulatum se nomme "barkeehi-debbi" (Piliostigma / femelle) et se distingue de Piliostigma thonningii désigné par "barkeehi-gorki" (Piliostigma / mâle) dont les feuilles sont pubescentes sur la face inférieure (AmBÉ \& Malaisse 2000). Par ailleurs, contrairement aux Malinké de Séguéla qui nomment les espèces sur la base des caractères botaniques de l'organe utilisé, combiné ou non à l'usage, les Peulh considèrent Gardenia ternifolia, à fruits comestibles, comme l'entité mâle "dii'aali-gorki" (Gardénia / mâle). L'espèce Gardenia erubescens, à fruits non comestibles, est l'équivalent femelle "dii'aali-debbi" (Gardenia / femelle). Ce dernier critère de dénomination ne se rencontre pas chez les Mossé du Burkina Faso.

La dénomination des espèces sur la base de leur milieu écologique s'apparente à celle adoptée par les Peulh du Cameroun. En effet, chez ces derniers, le "maayo", cours d'eau temporaire, donne son nom à l'espèce Ludwigia hyssopifolia "tabaahimaayo"(plante noire du maayo) (AMBÉ \& MALAISSE 2000; AMBÉ \& MALAISSE 2014). GuÈYE et al. (2006) considèrent que l'association du nom local de la plante à son habitat préféré est très souvent faite pour distinguer deux espèces proches ou qui ont une forte ressemblance mais ayant des habitats différents.

En outre, les noms de plante en malinké rapportés par GuÈYE et al. (2006) à Tomboronkoto dans le Sénégal Oriental (Région de Tambacounda) font référence à la morphologie (19,2\%), à un animal (19,2\%), à l'écologie ou habitat de l'espèce (11,5\%), à la dualité mâle/ femelle ou au sexe $(9,6 \%)$, à l'usage $(9,6 \%)$ et à une propriété de la plante $(5,7 \%)$. La référence à la morphologie est faite en rapport avec un caractère morphologique de la plante entière ou d'un de ses organes alors que le renvoi à un animal est le plus souvent dû à un lien écologique. Il en est également ainsi de l'évocation du sexe ou de la dualité mâle et femelle. Le plus souvent c'est un des usages les plus fréquents ou le plus populaire qui est associé au nom de la plante (GUÈYE et al. 2006).

Selon Silvano et Begossi (2002), Begossi et al. (2004), BeGossi et al. (2005), Souza \& Begossi (2006), Souza \& BEGOSSI (2007), GALVAGNE Loss et al. (2014), les populations de São Sebastião, de Rio de janeiro au Brésil utilisent des critères tels que la morphologie, l'écologie, l'usage, dans la taxonomie des espèces locales.

Ce processus permet de mettre au jour un trésor de savoirs locaux sur la biologie et l'écologie des espèces, ainsi qu'une foule de détails techniques ayant trait à la pêche (RUDDLE 1994; McClanahan et al. 1997; Neis et al. 1999; Johannes et al. 2000; Obura 2001; SABETIAN 2002).

\subsection{Variation des dénominations suivant les régions, ho- monymie des noms vernaculaires en langue Mooré}

Les noms vernaculaires sont pratiquement les mêmes dans les localités d'une même région, d'une même province administrative ou d'une même commune. Cependant, bien qu'appartenant à un même groupe ethnique, les noms vernaculaires varient largement d'une région à une autre. Les cas les plus emblématiques des noms vernaculaires sont: Kardega, Koumbrissaka, Widig-zaka, Zaguêm-sèega, Youniyounga, Boulli-Bissoum etc, respectivement attribués aux espèces: Acacia macrostachya, Cassia sieberiana, Stereospermum kunthianum, Albuca nigritana, Hiptis spicigera, Euphorbia hirta, dans les régions du nord et une petite portion de la région du sahel. Dans les régions du centre nord, du centre et du Plateau Central Mossé, ces espèces portent respectivement les noms vernaculaires Zamnèga, Yamtiiga, Ninyilinga, Boanga, Roungroungui, Walbissoum. Ces différences s'expliquent non seulement par la diversité des dialectes dans la langue locale Mooré mais aussi par la proximité avec d'autres groupes ethniques tels que les 
Gourmantché, les peulh, les Gourounsi, les Bisa, etc. Cette proximité peut influencer l'abandon d'un nom vernaculaire au profit d'autres. Ainsi, les habitants d'un village frontalier entre deux régions peuvent adopter deux appellations sur une même espèce.

\subsection{Perception des espèces}

Les perceptions paysannes de certaines espèces inventoriées confirment bien celles des études antérieures (BoGNOUNOU et al. 2001; SaVAdogo et al. 2011; Savadogo 2013). Ainsi, pour ces auteurs, les espèces comme Faidherbia albida, $\mathrm{Ca}$ lotropis procera, Stereospermum kunthianum, sont toutes frappées de mysticisme.

Deux attitudes sont possibles vis-à-vis de l'arbre, précise également GADOU (2001): une attitude profane lorsqu'on l'utilise pour des besoins énergétiques (cuisine, alimentation du bétail, teinture, etc.), une attitude religieuse lorsque ses parasites végétaux, ses racines, ses écorces ou ses feuilles participent à la transformation magique ou miraculeuse d'une situation quelconque. C'est cette dernière attitude que Coulibaly (1995) a appelé "prescription d'une liturgie qui transforme le végétal en autel". La solution à un problème qui touche les hommes peut se trouver dans l'observation de la nature (BAHUCHET et al. 1995; KYINDOU 2000). Cette perception se manifeste généralement en milieu rural par une association de phénomènes (expliqués et explicatifs) afin de montrer le mécanisme de réalisation des évènements. Ces associations sont d'autant plus aisées que dans la conception de l'homme traditionnel, il $\mathrm{y}$ a très peu de place au hasard (Bognounou et al. 2001; Savadogo 2013). Tout a un sens, les arbres et les phénomènes climatiques nous parlent. Ainsi, pour les populations animistes, l'arbre est un être capable de bien et de mal qui assure un lien entre l'individu et ses ancêtres (SAVAdogo 2008; SAVAdogo et al. 2010; Savadogo et al. 2011; Traoré et al. 2011; SAVADOGo 2013).

\section{Conclusion}

L'étude a permis de mettre en évidence diverses démarches utilisées en pays Mossé pour nommer et identifier les plantes. Les Mossé indigènes utilisent plusieurs critères pour les dénominations botaniques des espèces locales. Une étude ethnotaxonomique de bon nombre de ces espèces a permis de dénombrer 16 critères, allant de la morphologie foliaire à l'usage fait de la plante, en passant par les caractéristiques de l'espèce, son origine, son écologie, sa résistance aux stress hydrique, etc. Des similitudes d'approche avec d'autres groupes ethniques du Burkina Faso et d'ailleurs ont été mentionnées; quelques différences ont également été signalées. Soixante-douze (72) espèces au total ont été recensées. Ces espèces se répartissent en vingt-neuf (29) familles et en cinquante et un (51) genres. Les familles les plus dominantes sont les Commelinaceae et les Mimosaceae.

Les noms botaniques sont identiques dans les localités d'une même région tandis qu'ils varient d'une région à une autre selon les dialectes et la disponibilité des plantes de ladite localité. Certaines populations ont des perceptions positives des espèces végétales et de l'environnement qui les préserveraient d'exploitations multiples et multiformes par l'homme. Toutes ces connaissances et ces perceptions créées et perpétuées par les populations autochtones contribuent à une meilleure protection des espèces végétales en général et des espèces menacées et/ou en voie de disparition en particulier; d'où la nécessité de les valoriser et de les promouvoir afin d'encourager les populations à mieux préserver les communautés végétales.

\section{REMERCIEMENTS}

Nos premiers et sincères remerciements vont à l'endroit de toutes les populations en société Moagha qui ont accepté fournir toutes les informations sus mentionnées. Nous adressons également nos vifs remerciements à nos guides de terrain pour leurs sympathies et leurs franches collaborations.

\section{REFERENCES}

Ambé GA \& Malaisse F (2000): Diversité des plantes médicinales et ethnotaxonomie en pays malinké de Côte d'Ivoire, $2 \mathrm{p}$.

Ambé G A \& Malaisse F (2014): Les plantes utilisées dans la médecine et la pharmacopée traditionnelles d'une population malinké en Côte d'Ivoire. Revue Méd. Pharm. Afr. 14: 121-130.

Arbonnier M (2000): Arbres, arbustes et lianes d'Afrique de l'Ouest. 2ème édition, CIRAD-MNHN- UICN, 541 p.

Aubreville A (1936): La flore forestière de la Côte d'Ivoire. Paris, Larose, vol. 3, 208 p.

Bahuchet S, Grenant F, Grenant P, Joiris D, Pagezy H (1995): Etude comparative de la représentation symbolique des arbres et de la forêt équatoriale par quelques populations indigènes. Rapport d'activité pour la Commission européenne, DG XI, 6 p.

Begossi A, Castro F, Silvano R A M (2004): Ecologia humana e conservação. In Ecologia de pescadores da Mata Atlântica e da Amazônia Edited by: Begossi A. São Paulo: Ed. Hucitec; 313-324.

Begossi A, Clauzet M, Guarano L, Lima R V, Maccord P F, Ramires M, Silvano R A M (2005): Are biological species and high-ranking groups real? The ethnotaxonomy of fish on the Atlantic Forest coast of Brazil. In 20th Meeting of the Society for Human Ecology Salt Lake City, USA.

Berhaut J (1967): Flore du Sénégal. Dakar, Clairafrique, $485 \mathrm{p}$.

Bognounou O, Belem O M, Lamien N (2001): Connaissances et pratiques traditionnelles pour une conservation de la biodiversité au Burkina Faso, pp 105-122.

Bokdam J, Droogers AF (1975): Contribution à l'étude ethnobotanique des Wagenia de Kisangani (Zaïre), Wageningen, Institut National Agronomique, Pp. 1-74.

Coulibaly P B (1995): Rite et société à travers le Bafili: Une cérémonie d'initiation à la géomatie, chez les Bambara du Mali. Ed Jamana, Bamako, pp 31-32.

DUNCAN M (2005): La taxonomie vernaculaire des poissons de récif et l'intérêt du système participatif de suivi dans le Parc national de Wakatobi, sud-est de Célèbes (Indonésie). Ressources marines et traditions. Bulletin de la CPS 18:1835 .

EsOH E (2003): Plaider pour une protection des bois sacrés en Afrique noire. XII e congrès forestier mondial, Québec city, Canada, 6 p. 
FoALe S (1998): Que lire dans un nom? La taxonomie des poissons du Nggela occidental (Îles Salomon). Ressources marines et traditions, Bulletin de la CPS 9: 3-20.

GAdou M D (2001): Préservation de la biodiversité: les réponses des religions africaines, $19 \mathrm{p}$.

Galvagne Loss A T, Costa E M N, Machado C G, Flores F M (2014): Ethnotaxonomy of birds by the inhabitants of Pedra Branca Village, Santa Teresinha municipality, Bahia state, Brazil. Journal of Ethnobiology and Ethnomedicine 55: $1-15$.

GuÈYe M, AKPo L E, SAMB P I (2006): Etude ethnotaxonomique de quelques plantes de la Pharmacopée des Malinké de Tomboronkoto dans le Sénégal Oriental (Région de Tambacounda). Journal des Sciences et Technologies 4: 43-51.

JohanNes R, FreEman M, HAMilton R (2000): Ignore fishers' knowledge and miss the boat. Fish and Fisheries 1: 257-271.

KHOR M (2002): Intellectual Property, Biodiversity and Sustainable Development: Resolving the Difficult Issues. London, Zed Book, Penang, Third World Network, 104 p.

KYINDOU A A (2000): Culture et appropriation de l'information générale et spécialisée en milieu rural africain. HERMÈS 28, 11p.

Mcclanahan T, Glaesel H, Rubens J, Kiambo R (1997): The effects of traditional fisheries management on fisheries yields and the coral-reef ecosystems of southern Kenya. Environmental Conservation 24: 105-120.

MINISTÈRE DE L'ECONOMIE ET DU DÉVELOPPEMENT (2006): Atlas du Burkina Faso, 215 p.

Neis B, Schneider D C, Felt L, Haedrich R L, Fischer J, Hutchings J A (1999): Fisheries assessment: what can be learned from interviewing resource user? Canadian Journal of Fisheries and Aquatic Science 56: 1949-1963.

OBURA D O (2001): Participatory monitoring of shallow tropical marine fisheries by artisanal fishers in Diani, Kenya. Bulletin of Marine Sciences 69: 777-791.

RudDLE K (1994): Local knowledge in the future management of inshore tropical marine resources and environments. Nature and Ressources 30: 28-37.

Sabetian A (2002): L'importance des connaissances ethnographiques dans l'élaboration et la gestion de projets de recherche halieutique dans le Pacifique Sud: étude faite dans l'île de Kolombangara (Îles Salomon). Ressources marines et traditions, bulletin de la CPS 14: 22-34.

SAVADOGo S (2008): Etude de la flore et de la végétation des bois sacrés de la zone sub-sahélienne du Burkina Faso. Mémoire de DEA, Université de Ouagadougou, 65 p.

Savadogo S (2013): Les bois sacrés du Burkina Faso: diversité, structure, dimension spirituelle et mode de gestion de leurs ressources naturelles. Thèse de doctorat unique, Université de Ouagadougou (Burkina Faso), 280 p.

Savadogo S, Oú́draogo A, Thiombiano A (2010): Perceptions, mode de gestion et végétation des bois sacrés au nord du Burkina Faso. Flora et Vegetatio Sudano-Sambesica 13: $10-21$.

Savadogo S, Ouédraogo A, Thiombiano A (2011): Diversité et enjeux de conservation des bois sacrés en société Mossi (Burkina Faso) face aux mutations socioculturelles actuelles. Int. J. Biol. Chem. Sci. 5: 1639-1658.
Silvano R, Begossi A (2002): Ethnoichthyology and fish conservation in Piracicaba River, Brazil. Journal of Ethnobiology 22: 285-306.

Souza S P, Begossi A (2006): Ethnobiologia de Sotalia fluviatilis (Gervais, 1853) no litoral norte do estado de São Paulo, Brasil. Workshop on Research and Conservation of the Genus Sotalia, Rio de Janeiro, 39 p.

Souza S P, Begossi A (2007): Whales, dolphins or fishes? The ethnotaxonomy of cetaceans in São Sebastião, Brazil. Journal of Ethnobiology and Ethnomedicine 9: 1-15.

Spichiger R E, Savolanen V V, Figeat M (2000): Botanique systématique des plantes à fleurs. Une approche phylogênétique nouvelle des Angiospermes des régions tempérées et tropicales, Lausanne (Suisse), Presses Polytechniques et Universitaires Romandes, 372 p.

Traoré L, Ouédraogo I, Ouédraogo A, Thiombiano A (2011): Perceptions, usages et vulnérabilité des ressources végétales ligneuses dans le Sud-Ouest du Burkina Faso. Int. J. Biol. Chem. Sci. 5: 258-278.

Yahmed B D (2006): Atlas du Burkina. Edition J.A., Paris, $54 \mathrm{p}$.

YANN LE GOATER (2007): La protection des savoirs traditionnels: l'expérience indienne. Séminaire Jeunes Chercheurs - Association Jeunes Études Indiennes, 16 p. 